\title{
MULHERES INTERNADAS POR AGRESSÃO EM UM HOSPITAL DE PRONTO SOCORRO: (in)visibilidade da violênciaa
}

\author{
M ichele M azza IL HA ${ }^{b}$, Sandra M aria Cezar LEAL' , Joannie dos Santos F achinelli SOA RE $S^{d}$
}

\section{RESUM 0}

T rata-se de um estudo do tipo transversal, com o objetivo de caracterizar a hospitalização de mulheres vítimas de agressão, no Hospital de Pronto Socor ro de Porto A leg re, R io G rande do Sul, durante o ano de 2005. A col eta dos dados foi realizada nos prontuários de mulheres com idade a partir de 18 anos, vítimas de agressão. $A$ análise foi descrita a partir da utilização do Software E pi-I nfo, com a categorização dos eventos, do cruzamento de variáveis descritivas e de índices frequenciais absolutos e relativos. No período do estudo, foram hospital izadas 73 mul heres, vítimas de ag ressão; 49,3\% na faixa etária de 18 a 29; 41,1\% foram ag redidas com arma de fogo e 37\% com arma branca. $\mathrm{N}$ a maioria dos prontuários, os dados estavam incompletos e/ ou faltavam registros, o que pode indicar que muitos casos de agressões não foram identificados durante o período da hospitalização e que o tratamento ficou restrito às lesões físicas.

D escritores: Violência contra a mulher. G ênero e saúde. H ospitalização.

\section{RESUMEN}

Se trata de un estudio transversal con el objetivo de caracterizar a la hospitalización de las mujeres víctimas de agresión en el H ospital deP rimer os Socor ros de P or to A legre, R io G rande do Sul, B rasil , en 2005. L a recol ección del os datos fue realizada en los prontuarios de mujeres con más de 18 años, víctimas de agresión. E I análi isis fuedescrito, a partir dela utilización del Software E pi-Info, con la categorización de los eventos y cruz ando variables descriptivas eíndices frecuenciales absolutos y relativos. E n el período del estudio, fueron hospitalizadas 73 mujeres, víctimas de agresión; 49,3\% en la edad de 18 a 29; $41,1 \%$ fueron agredidas con arma de fuego y $37 \%$ con arma blanca. E n la mayoría de los prontuarios, los datos estaban incompletos y/ o faltaban registros, lo que puede indicar que muchos casos de agresiones no fueron identificados durante el período de la hospitalización y que el tratamiento quedó restricto a las lesiones físicas.

D escriptores: Violencia contra la mujer. G énero y salud. H ospitalización.

T itulo: M ujeres internadas por agresión en un hospital de emergencia: (in)visibilidad de la violencia.

\section{ABST RACT}

This is a cross-sectional study that aims to characterize the hospitalization of femal evictims of aggression at $\mathrm{H}$ ospital de P ronto Socor ro de P orto A legre, R io G rande do Sul, B razil, over the year 2005. The data collection was done through the analysis of the records of women above 18 years old who had been victims of aggression. The analysis was described through the use of E pi-Info Software, with the categorization of the events and the crossing of descriptive variables and absolute and relative frequency indexes. 0 ver the studied time frame, 73 women, victims of aggression, enter ed the hospital; $49,3 \%$ of them were betw een the ages of 18 and $29 ; 41,1 \%$ were assaulted with firearm and $37 \%$ with white w eapon. In nearly all records, data were incomplete or lacked registrations at all, an indication that many cases of aggression were not identified during the hospitalization time and that the treatment was restricted to physical lesions.

D escriptors: Violence against women. G ender and health. H ospitalization.

T itle: Women interned due to aggression at an emergency hospital: the (in)visibility of violence.

\footnotetext{
aE xtraído da monografia apresentada em 2007 ao Curso de G raduação em E nfermagem da E scola de E nfermagem da U niversidade do Vale do Rio dos Sinos (U nisinos).

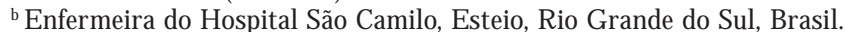

c D outoranda em E nfermagem, Professora da U nisinos, São L eopoldo, Rio Grande do Sul. Brasil.

a Enfermeira, M estranda em Enfermagem pela Escola de Enfermagem da U niversidade Federal do Rio Grande do Sul (UFRGS), Porto Alegre, Rio Grande do Sul, Brasil.
} 


\section{INT RODUÇÃO}

A violência contra a mulher é um fenômeno que revela as desigual dades entre homens e muIher es na sociedade, "é uma das violações de direitos humanos mais praticadas e menos reconhecidas no mundo"(1). T rata-se de um problema de saúde e de Saúde Pública(2), pois afeta a integ ridade corporal e o estado psíquico e emocional da vítima, acarretando sérias e graves consequências para 0 seu pleno e integral desenvolvimento, comprometendo-Ihe o exercício da cidadania e dos direitos humanos ${ }^{(3)}$.

A pesquisa realizada intitulada "A mulher brasileira no espaço público e privado", constatou que $43 \%$ das mulheres já sofreram algum tipo de violência sexual, sendo que mais de $50 \%$ não pediram ajuda e em $53 \%$ dos casos, os maridos e parceiros foram os agressores. A ponta ainda que uma em cada cinco brasileiras declara espontaneamente já ter sofrido algum tipo de violência por parte de um homem e, a cada 15 segundos, uma mulher é espancada por um homem, no Brasil(4).

Tendo em vista as estatísticas cada vez mais al armantes sobre a violência contra mulheres, considera-se necessário conhecer a população feminina atendida em um hospital de trauma, em decorrência da agressão, bem como os principais fatores envolvidos. N esse contexto, a equipe de saúde pode ter papel importante na visibilização do atendimento dessas mulheres, quando chegam às emergências, vítimas de algum tipo de violência. Ressal ta-se que, em muitos casos, esta é uma das únicas oportunidades que elas têm para romper 0 silêncio que envolve as situações de violência, em especial a perpetrada por parceiro íntimo. Silêncios "reforçados quer pela própria mulher que a vive, quer pelos profissionais que eventualmente ela procura"(5). Salienta-se que a inter nação em um hospital de pronto socorro só ocorre quando o trauma físico é grave o que indica que a mulher pode estar correndo risco de morte.

N o Brasil, ainda são precários os registros e a notificação nos serviços de saúde, dos atendimentos às usuárias em situação de violência, dentre os vários motivos, o principal é a naturalização e banalização da violência contra a mulher, que é percebida pelo sendo comum como algo normal(6), também destaca-se 0 "desconhecimento dos profissionais de saúde sobre as consequências do evento para a saúde da mulher, [ ...] e por não se sentirem preparados para lidar com a situação, considerando-a um problema de cunho familiar e não vendo necessidade de realizar a notificação"(7).

$\mathrm{O}$ intuito de investigar o tema originou a $\mathrm{M}$ 0nog rafia apresentada ao Curso de G raduação em Enfermagem da Escola de Enfermagem da U niversidade do Vale do Rio dos Sinos, da qual apresenta-se alguns resultados(8). A ssim, 0 objetivo do estudo foi caracterizar a hospitalização de mulheres vítimas de agressão, em um hospital público de trauma, no período de janeiro a dezembro de 2005.

\section{METODOLOGIA}

T rata-se de um estudo exploratório, descritivo, do tipo transversal, desenvolvido no Hospital de Pronto Socorro de Porto Alegre, Rio Grande do Sul.

Os dados foram coletados nos prontuários de mulheres com idade a partir de 18 anos, hospital izadas por agressão, no período de janeiro a dezembro de 2005. Para a identificação dos casos foi solicitada ao setor de Documentação e Estatística do H ospital a listagem dos prontuários das mulheres internadas, cuja causa estivesse relacionada à agressão e ferimento (por arma branca, por arma de fogo, ag ressões outras). Foram excluídas as mulheres que após a aval iação dos prontuários, havia a informação que o ferimento se deu acidentalmente. Os ferimentos por arma branca e arma de fogo podem estar relacionados à agressão, entretanto, só foram incluídas as mulheres em que, no prontuário, havia algum registro dos profissionais da Saúde constatando a agressão.

A análise foi descrita a partir da utilização do software E pi-Info pela categorização dos eventos, pelo cruzamento de variáveis descritivas e de índices frequenciais absolutos e relativos.

E mbora não se trate de pesquisa com seres humanos, o projeto foi enviado para apreciação ao Comitê de Ética em Pesquisa da Secretaria M unicipal de Saúde de Porto Alegre para utilização de dados de prontuário, tendo sido aprovado (registro 63, processo no 001.03345.06.2.6).

\section{APRESENTAÇÃO DOS DADOS E DISCUSSÕES}

No período de janeiro a dezembro de 2005, foram internadas no Hospital de Pronto Socorro (HPS) 73 mulheres com idade a partir de 18 anos, vítimas de agressão. 
$\mathrm{N}$ a distribuição das mulheres inter nadas por agressão quanto à faixa etária (T abela 1 ), identificou-se que as internações decresceram com 0 aumento da idade, indicando que as mulheres mais jovens estão mais expostas às agressões.

T abela 1 - Distribuição das mulheres internadas por agressão no HPS, no ano de 2005, quanto à faixa etária, local do corpo lesado e tipo de agressão. Porto Alegre, RS, 2007.

\begin{tabular}{|c|c|c|}
\hline Variáveis & $\mathbf{n}$ & $\%$ \\
\hline \multicolumn{3}{|l|}{ Faixa E tária } \\
\hline 18 a 29 anos & 36 & 49,3 \\
\hline 30 a 39 anos & 14 & 19,2 \\
\hline 40 a 49 anos & 13 & 17,8 \\
\hline 50 a 59 anos & 7 & 9,6 \\
\hline$M$ ais de 60 anos & 3 & 4,1 \\
\hline T otal & 73 & 100,0 \\
\hline \multicolumn{3}{|l|}{ Local do corpo lesado } \\
\hline Tórax/ abdômen & 25 & 34,2 \\
\hline $\begin{array}{l}\text { M embros superiores/ membros in- } \\
\text { feriores }\end{array}$ & 15 & 20,5 \\
\hline Tórax/ abdômen e/ ou M sSs/ M sls & 9 & 12,3 \\
\hline Cabeça & 6 & 8,2 \\
\hline Face & 6 & 8,2 \\
\hline F ace e cabecca & 4 & 5,5 \\
\hline M sSs/ face/ coluna cervical & 4 & 5,5 \\
\hline Coluna lombar/ face & 3 & 4,2 \\
\hline Cabeça/ M sSs & 1 & 1,4 \\
\hline T otal & 73 & 100,0 \\
\hline \multicolumn{3}{|l|}{ T ipo de agressão } \\
\hline Agressão por arma de fogo & 30 & 41,0 \\
\hline A gressão por arma branca & 27 & 37,0 \\
\hline Agressão física & 9 & 12,3 \\
\hline Agressão física com objetos & 4 & 5,5 \\
\hline Outros & 3 & 4,2 \\
\hline T otal & 73 & 100,0 \\
\hline
\end{tabular}

Legenda: M SSs: membros superiores; M sls: membros inferiores.

Em pesquisa realizada, no mesmo hospital, sobre internações por violência, identificou-se que os homens representaram $90,5 \%$ da população em estudo. Porém, para as mulheres a faixa etária de 10 a 19 anos representou $25,7 \%$, enquanto a mesma faixa etária, para os homens correspondeu a $17,3 \%$ das internações ${ }^{(9)}$. Resultados que corroboram com estudo realizado em unidades de emer- gência credenciadas ao Sistema Ú nico de Saúde (SU S), no D istrito Federal, com atendimentos decorrentes de violência, no qual a faixa etária de 10 a 19 anos representou 21,1\% para as mulheres e $19,4 \%$ para os homens ${ }^{(10)}$.

A violência doméstica atinge, principalmente, mulheres na faixa de idade produtiva e reprodutiva, resultando, daí, significativos índices de incapacidade ou morte, como, por exemplo, o de que um em cada cinco dias de falta ao trabal ho ocorre em decorrência da violência doméstica sofrida por mulheres ${ }^{(3)}$.

Quanto ao local do corpo lesado (T abela 1), os membros superiores e inferiores, quando associados aos traumas de tórax/ abdômen, face, coluna cervical e cabeça são o segundo local do corpo mais lesado pela ag ressão. As lesões na parte superior do corpo (face, pescoço e braços), demonstram, não só a tentativa de proteger-se da agressão, por parte da vítima, mas também a intenção do agressor em humilhar e agredir a dignidade da mulher ${ }^{(11)}$.

$\mathrm{N}$ as mulheres, os traumas torácicos, de face (relacionados ou não ao traumatismo crânioencefálico), bem como os traumatismos múltiplos, foram os diagnósticos de maior frequência, em outro estudo realizado, no mesmo hospital, com pacientes internados por agressão, tal diagnóstico foi frequente em mulheres hospitalizadas na U nidade de T ratamento Intensivo (UTI) de trauma, que tiveram lesões graves, com maior possibilidade de óbito(12).

A face é 0 alvo preferido dos agressores, sendo freqüentes as lesões de olhos e mandíbula e, em menor número de casos, ocorrem chutes como forma de agressão. De acordo com o relatado, as lesões de mãos e braços ocorrem quando a mulher tenta proteger-se das ag ressões no rosto ${ }^{(13)}$.

A T abela 1 apresenta-se os tipos de agressões, identificadas nos prontuários. $N$ as agressões físicas, estão incluídas vandalismo, brigas interpessoais relacionadas ao uso de drogas e brigas entre casais. A categoria "outros" corresponde a 1 caso de estupro e a de 2 mulheres vítimas de queimaduras, que se tem suspeita de ag ressão. E ntretanto, os dados registrados nos prontuários sobre as circunstâncias em que ocorreram as queimaduras não for neceram informações para que se possa incluir como agravo intencional. Vale dizer que essa lacuna contribui para aumentar a invizibilização da violência contra a mulher tanto nos serviços de Saúde, quanto na sociedade e no censo comum. 
Com relação ao motivo da agressão, conforme observa-se na T abela 2, deparou-se com a falta de informação, pois em aproximadamente metade das ocorrências, esse dado não foi registrado.

T abela 2 - Distribuição das mulheres inter nadas por agressão no HPS, no ano de 2005, quanto ao motivo da agressão; relação com o agressor; e local de ocorrência. Porto Alegre, RS, 2007.

\begin{tabular}{lrr}
\hline Variáveis & $\mathbf{n}$ & $\%$ \\
\hline M otivo da agressão & & \\
A ssalto & 11 & 15,1 \\
Briga interpessoal & 4 & 9,6 \\
Separação & 14 & 19,5 \\
Outros & 37 & 50,7 \\
Não informado & $\mathbf{7 3}$ & $\mathbf{1 0 0 , 0}$ \\
\hline T otal & & \\
\hline Agressor(a) & 13 & 17,8 \\
D esconhecido & 9 & 12,3 \\
Outra mullher & 7 & 9,6 \\
Companheiro & 2 & 2,7 \\
F amiliar & 1 & 1,4 \\
T entativa de suicídio & 41 & 56,2 \\
Não informado & $\mathbf{7 3}$ & $\mathbf{1 0 0 , 0}$ \\
\hline T otal & & \\
\hline Local de ocorrência & 16 & 21,9 \\
Via pública & 8 & 11,0 \\
Casa & 3 & 4,1 \\
Via pública (próximo casa) & 1 & 1,4 \\
T rabalho & 45 & 61,6 \\
Não informado & $\mathbf{7 3}$ & $\mathbf{1 0 0 , 0}$ \\
\hline T otal & & \\
\hline
\end{tabular}

Os resultados deste estudo não corroboram com o de outras pesquisas, como a do Instituto Patrícia Galvão, na qual $81 \%$ dos entrevistados apontam o uso de bebidas alcoólicas como fator desencadeante de ag ressões contra mulheres; 37\% o desemprego; $31 \%$ problemas com dinheiro; $14 \%$ dificuldades no trabalho, e até mesmo a falta de comida em casa, com $13 \%$, como justificativa para a agressão(14).

Em pesquisa realizada em Salvador, Bahia, com 701 mulheres $86,6 \%$ das entrevistadas declararam não existir nenhum motivo para a agressão, sendo que $13,4 \%$ apresentaram como principais motivos a infidelidade feminina e a não realização dos trabal hos domésticos de for ma satisfatória, no entendimento de seu companheir ${ }^{(15)}$. Portanto, registrar os motivos pelos quais ocorreu a agressão pode contribuir para a visibilização da violência contra a mulher e gerar mais subsídios para a elaboração de ações preventivas e de enfrentamento à violência.

N este estudo, os dados relativos à caracterização do agressor também estão comprometidos. Diante dessa condição levantam-se as seguintes indagações: 0 agressor era desconhecido? N ão foi fornecido na infor mação porque a paciente omitiu o dado ou não foi solicitada? 0 não registro foi decorrente da desconsideração por esse tipo de agravo, desinformação por parte dos profissionais envolvidos, quanto à importância da informação, ou ainda, pela banalização da violência contra a muIher?

Não é intenção responder a tais questionamentos neste estudo, entretanto propõe-se reflexão sobre o tema, pois cabe ressaltar que este dado é importante, pois, sem essa informação, não há como responsabilizar o companheiro íntimo pela agressão. A lém disso, considera-se que saber por quem a mulher foi ag redida, é necessário, tanto para fazer a notificação e visibilizar o tipo de violência, orientar a importância de registrar queixa na Delegacia da M ulher, quanto para realizar um atendimento de Saúde Pública, voltado ao esclarecimento/ encaminhamento da paciente aos serviços de violência contra a mulher existentes na cidade, e se for caso, possibilitar que, após a alta hospitalar, a paciente não tenha que retornar para o "convívio" com o agressor.

0 risco de agressão às mulheres é maior por parte de pessoas próximas, como parceiros e familiares, do que por estranhos, sendo que oscilam entre $20 \%$ e $50 \%$ o total de mulheres, ao redor do mundo, agredidas, pel o menos uma vez na vida, por seus companheiros. No Brasil, estes índices são apurados, basicamente, por meio dos casos denunciados nas delegacias de defesa da mulher e atingem 0 patamar elevado de $77,6 \%{ }^{(10)}$. N as mulheres hospitalizadas por agressão, a maior parte dos traumas é praticada por companheiros ou ex-companheiros, e mesmo quando o agressor não consta nos registros, as vítimas acabam por denunciá-los à equipe de enfermagem ${ }^{(12)}$.

E studo realizado em outro serviço de urgência e emergência com usuárias em situação de violência revelou que $44 \%$ das mulheres deixaram a 
casa onde residiam com seus agressores. Entretanto, $46,5 \%$ retor naram para o convívio com o mesmo, sendo que $20 \%$ al egaram ter voltado por causa dos filhos e $29,6 \%$, após o agressor prometer não mais agredir ${ }^{(15)}$

A equipe de saúde tem conhecimento de que a mulher, vítima de violência, quando procura socorro médico, sente muita vergonha, medo e constrangimento em revelar a origem de suas lesões. Cerca de 35\% das atendidas nas emergências com lesões, quando abordadas de maneira adequada pelos profissionais, admitem ter sofrido violência ${ }^{(13)}$.

$\mathrm{N}$ essa perspectiva, 0 envolvimento da equipe de saúde treinada para dar suporte à mulher em situação de violência propicia a identificação dos casos na demanda dos atendimentos, com vistas ao auxílio para romper com a relação violenta, à orientação e ao incentivo a buscar ajuda em delegacias de mulheres, casas de apoio, abrigos, entre outros, além de conquistar a confiança dessa usuária, que se encontra frágil e com medo do que possa acontecer, caso relate ser vítima de agressão(9). Considera-se que, nesse contexto, abster-se de qualquer espécie de comentário sobre o ocorrido e voltar-se unicamente para o bem-estar da paciente, pode contribuir para que ela se sinta à vontade e relate 0 que achar necessário.

Entretanto, resultados de estudo realizado com mulheres abrigadas na Casa de A poio Viva $\mathrm{M} \mathrm{a-}$ ria, em Porto Alegre, apontaram que a maiorias das que haviam buscado 0 atendimento nos serviços básicos de saúde tiveram dificuldades para expressar a situação de violência; muitas não foram reconhecidas como mulheres em tal situação, exceto quando verbalizavam aos profissionais de saúde. N essa perspectiva, "o problema da violência fica oculto, e o tratamento se encaminha para os sintomas que compreendem desde pal pitações, ansiedade, insônia e alterações digestivas, decorrentes desse contexto"(16).

Portanto, o envolvimento dos serviços básicos de saúde, com investimento e qualificação dos profissionais na detecção da violência contra a mulher, pode evitar os casos graves que chegam às emergências e interferir na prevenção de fatalidades. Salienta-se ainda que os profissionais assumem posição estratégica em todo o país, por atenderem diariamente, mulheres que buscam os serviços de saúde.

O utro dado que destaca-se neste estudo é que a via pública correspondeu ao primeiro local da agressão identificado, o que não corrobora com resultados de outros estudos que constataram que as mulheres estão mais vulneráveis à ag ressão no espaço doméstico do que na via pública ${ }^{(10-12)}$.

Considerou-se relevante pesquisar nos prontuários das mulheres em estudo, informações sobre o dia da semana em que ocorreu a hospitalização, para tentar identificar se havia al guma relação com finais de semana e elencar mais dados para contribuir com a análise, no entendimento de como e porque acontecem essas agressões. Identificou-se que as agressões foram mais frequentes nos finais de semana, pois a soma de sexta, sábado e domingo correspondeu a $54,8 \%$ dos casos de mulheres hospitalizadas por agressão. Pode-se inferir que esses três dias da semana estão relacionados a festas, uso de bebidas e drogas, ou ao fato de o casal passar mais tempo juntos. A quartafeira representou o percentual de $13,7 \%$, dia da semana que é considerado, por alguns, como "o dia de namorar". Entretanto, como não dispomos de dados referentes ao cotidiano das mulheres em estudo, não se pode inferir que essas práticas culturais possam estar relacionadas à frequência das agressões, nesses dias.

Os dados desta pesquisa corroboram, em parte, outro estudo realizado, uma vez que os dias da semana que mais apareceram foram domingo (24\%), quarta-feira (24\%) e terça-feira (19\%), no H ospital M unicipal M iguel Couto e, segunda-feira (23,5\%), sábado (19,6\%), domingo (17,6\%) equarta-feira (14\%), como os dias mais procurados no Hospital M unicipal Salgado Filho ${ }^{(13)}$.

Considera-se que saber os dias da semana nos quais ocorrem com maior frequência os atendimentos de mulheres em situação de violência pode indicar a escolha de tais dias, para pesquisadores interessados em realizar pesquisas qualitativas, entrevistar essas mulheres, bem como servir de alerta para os profissionais na identificação dos casos decorrentes de agressão.

Pelas informações nos prontuários relacionadas à procedência das mulheres internadas por agressão, no H ospital em estudo, identificou-se que a maioria era proveniente da cidade de Porto A legre, 49 (67,1\%), o que éjustificado pela local ização do Hospital de Pronto Socorro, entretanto, 17 $(23,3 \%)$ das internações foram oriundas de cidades da G rande Porto Alegre, possivelmente encaminhadas ao Hospital de Pronto Socorro devido à gravidade do trauma causado pela agressão. Ob- 
serva-se, também, que as vítimas provenientes de outros locais que não Porto Alegre, foram encaminhadas ao HPS em função da gravidade de seu estado, isto é, foram atendidas em hospitais de sua região e, na falta de recursos ou médicos especializados, foram transferidas para a capital.

No intuito de identificar para onde a mulher hospital izada por agressão é encaminhada/ retorna após a alta hospitalar, pesquisou-se a informação nos prontuários, utilizando-se a palavra destino para se referir à destinação da usuária, constatando-se que $50(68,5 \%)$ mulheres voltaram para suas casas e $14(19,2 \%)$ foram transferidas para outros hospitais. Os critérios para a transferência são a complexidade do trauma e o usuário ter plano de saúde privado.

Não foi possível identificar para onde $6,8 \%$ das mulheres foram após a alta hospitalar, pois não havia o registro sobre seus destinos. 0 percentual de óbitos decorrentes das internações por agressão foi de 5,5\% mulheres, sendo que os tipos de agressão foram por arma de fogo, arma branca e outros.

N os registros dos prontuários não foi possível identificar se houve encaminhamento das muIheres hospitalizadas por agressão, para serviços de referência, como exemplo, casa abrigo e delegacia da mulher. T ambém não foi possível saber se as mulheres retornaram a residir ou não com 0 agressor, pois não havia registros sobre tal informação.

Resultados de outra pesquisa realizada no hospital em estudo também relatam dificuldades com a falta de registros, o que gera questionamentos sobre o local onde as mulheres foram agredidas, se tinham residência fixa, se chegaram sozinhas ao hospital e sem condições de informar 0 endereço pela gravidade do trauma. "O u, ainda, se o dado não foi registrado por não ser considerado uma informação importante, diante da queixa clássica de assalto"(9).

N esse contexto, aponta-se ainda, que no Brasil, muitas das informações sobre 0 atendimento de usuários vítimas de violência, ainda continuam invisibilizados e inacessíveis para gestores e responsáveis por decisão pelas políticas de saúde pública, "seja pelo atual modelo de vigilância, seja pela intrincada rede de fatores que favorecem o silêncio acerca da temática violência, principalmente quando as vítimas são mulheres, crianças e idosos"(10).

Considera-se que os registros completos relacionados aos atendimentos e hospital izações de mulheres, em decorrência de agressões, auxiliam a visibilizar e combater a banalização desse tipo de violência. No entanto, o dado sobrea violência contra a mulher é pouco investigado pelos profissionais e Serviços de Saúde e, em geral, só é evidenciado quando a mulher opta por informar tal situação. Porém, na maioria dos casos, isso não acontece e muitos dos atendimentos decorrentes de violência não são identificados nos Serviços Púbicos de Saúde.

\section{CONCLUSÕES}

I dentificou-se que no período de janeiro a dezembro de 2005 , foram internadas no hospital em estudo 73 mulheres com idade a partir de 18 anos, vítimas de ag ressão e a faixa etária de 18 a 39 anos, representou $68,5 \%$ das internações. Os membros superiores e inferiores, associados aos traumas de tórax/ abdômen, face, coluna cervical e cabeça foram o segundo local do corpo mais lesado, caracterizando o tipo de agressão sofrida pelas muIheres em situação de violência. As agressões foram causadas por arma de fogo, arma branca, agressão física e/ ou com objetos, entre outras.

Deparou-se com a falta de registros nos prontuários sobre o agressor, bem como, os motivos da violência. Fato que contribuí para a invisibilização deste tipo de agravo, principalmente, envolvendo parceiro íntimo.

Os registros incompletos e/ ou inexistentes impossibilitaram uma leitura mais detalhada sobre o tema. Ressalta-se que esta é uma forma do Serviço de Saúde expressar o descaso e o não reconhecimento desse tipo de agravo, ou seja, a banalização da violência contra a mulher e a sua não inclusão como um problema de Saúde Pública.

Enfatiza-se que a internação de mulheres em um hospital de trauma representa a gravidade desse tipo de violência, pois em muitas situações ficam restritas ao ambiente doméstico e não evidenciadas, ou seja, as que chegam às emergências, além de serem sobreviventes, têm traumas físicos graves, estão enfrentando situações de violência a muito mais tempo do que se pode imaginar e, correm risco de morte, pois as próximas agressões poderão ser fatais.

Considera-se importante que os processos de trabalho dos serviços Públicos de Saúde estejam voltados à integralidade do atendimento às usuárias, proposto como diretriz do SUS, no qual 0 
atendimento deve ir além da lesão física ou problema orgânico, no caso das mulheres em situação de violência. Nesse sentido, as redes de proteção e redes sociais para enfrentamento da violência devem estar articuladas com os serviços e os profissionais de saúde em condições de enfrentar as situações e possibilitar que essas usuárias tenham a oportunidade de sair da situação de violência e ressignificar suas vidas.

\section{REFERÊNCIAS}

1 Grossi PK. Violência contra a mulher: complicações para os profissionais. In: Lopes MJM, M eyer DE, Waldow VR. G ênero e saúde. Porto Alegre: Artes M é dicas; 1996. p. 133-49.

2 Schraiber LB, D' Oliveira AFPL, Couto M T. Violência e saúde: contribuições teóricas, metodológicas e éticas de estudos da violência contra a mulher. Cad Saúde Pública. 2009;25 Supl 2:s205-16.

3 Dagord ALL. Viva M aria: dez anos [ dissertação] . Porto Alegre: E scola de E nfermagem, U niversidade Federal do R io G rande do Sul; 2003.

4 Venturi G, Recamán M , Oliveira S. A mulher brasileira no espaço público e privado. São Paulo: F undação Perse A bramo; 2004.

5 Schraiber LB, D' Oiveira AFPL. Romper com a violência contra mulher: como lidar desde a perceptiva do campo da saúde. In: M eneguel SN, organizador. Rotas críticas II: ferramentas para trabal har com a violência de gênero. Santa Cruz do Sul: EDUN ISC; 2009. p. 133-41.

6 Silva RC, A Imeida R M , Costa T CR. Violência contra a mulher: um estudo com mulheres membros do Clube de $M$ ães do bairro do José Pinheiro em Campina G rande - PB. In: A nais do 13을 ncontro Latino A mericano de Iniciação Científica, 9o Encontro L atino A mericano de Pós-Graduação; 2009 out 16-17; São José dos Campos, B rasil [ I nternet] . Jacareí: U niversidade do Vale do Paraíba; 2009 [ citado 2010 fev 26] . Disponível em: http:/ / www.inicepg.univap.br/cd/ INIC_2009/ anais/ arquivos/0895_0459_01.pdf.

\section{Endereço da autora / Dirección del autor / Author's address:}

Joannie dos Santos F achinelli Soares

Rua Primeiro de M aio, 410, Partenon

90660-190, Porto Alegre, RS

E-mail:jofachi@hotmail.com
7 O kabe I, Fonseca R M GS. Violência contra a mulher: contribuições e limitações do sistema de infor mação. Rev Esc Enferm USP. 2009;43(2):453-8.

8 Ilha M M . A (in)visibilidade da violência contra a muIher: mulheres internadas por agressão em um hospital de pronto socorro [ monografia]. São L eopoldo: U niversidade do Vale do R io dos Sinos; 2007.

9 L eal SM C, Lopes M J M . Violência como objeto da assistência em um hospital de trauma: o "olhar" da enfermagem. Ciênc Saúde Colet. 2005;10(2):419-31.

10 M ascarenhas M D M, Silva M M A, M alta D C, M oura L, M acário EM, Gawryszewski VP, et al. Perfil epidemiológico dos atendimentos de emergência por violência no Sistema de Serviços Sentinel as de V igilância de Violências eA cidentes (V iva) - Brasil, 2006. E pidemiol Serv Saúde. 2009;18(1):17-28.

11 Schraiber LB, D'Oliveira AFPL, França-J unior I, Pinho AA. Violência contra a mulher: estudo em uma unidade de atenção primária à saúde. Rev Saúde Pública. 2002;36(4):470-7.

12 L eal SM C, L opes M J M . M as allá del traumas: mujeres hospitalizadas por agresiones. Rev Panam Enferm. 2005;3(1):32-40.

13 Deslandes SF, G omes R, Silva CM F P. Caracterização dos casos de violência doméstica contra a muIher atendidos em dois hospitais públicos do Rio de Janeiro. Cad Saúde Pública. 2000;16(1):129-37.

14 Jordão F P. Percepções e reações da sociedade sobre violência contra a mulher: análise de pesquisa: pesquisa I bope/ Instituto Patrícia Galvão (2006) [ Internet]. São Paulo: Instituto Patrícia Galvão: 2006 [ citado 2010 fev 26]. Disponível em: http:/ / www.agenciapatriciagalvao.org.br/images/ stories/ PD F / pesquisas/ pesq_ibope_2006.pdf.

15 Silva IV. Violência contra mulheres a experiência de usuárias de um serviço de urgência e emergência de Salvador, Bahia, Brasil. Cad Saúde Pública. 2002; 19(2):263-72.

16 Riquinho D L, Correia SG . 0 papel dos profissionais de saúde em casos de violência doméstica: um relato de experiência. Rev G aúcha Enferm. 2006;27(2):301-30.

Recebido em: 11/ 03/ 2010

A provado em: 19/ 05/ 2010 